UB-ECM-PF-99/01

KUL-TF-99/5

UTTG-01-99

hep-th/9901060

\title{
BPS solutions of a D5-brane worldvolume in a D3-brane background from superalgebras
}

\author{
Ben Craps ${ }^{+, 1}$, Joaquim Gomis ${ }^{*, \dagger, 2}$ \\ David Mateos ${ }^{*, 3}$ and Antoine Van Proeyen ${ }^{+, 4}$ \\ * Departament ECM, Facultat de Física \\ Universitat de Barcelona and Institut de Física d'Altes Energies, \\ Diagonal 647, E-08028 Barcelona, Spain \\ + Instituut voor theoretische fysica, \\ Katholieke Universiteit Leuven, B-3001 Leuven, Belgium \\ $\dagger$ Theory Group, Physics Department \\ University of Texas at Austin \\ Austin, TX 78712 USA
}

\begin{abstract}
The BPS method is used to find BPS solutions of the worldvolume theory of a D5brane in the near horizon geometry of a D3-brane. The BPS bound is interpreted in terms of the 'maximally extended' D5 worldvolume supersymmetry algebra in the corresponding curved background, which is $O S p(1 \mid 16)$. This algebra is an extension of the worldvolume superalgebra $O S p\left(4^{*} \mid 4\right)$. The analysis is generalized to the non-near horizon case.
\end{abstract}

\footnotetext{
1 Aspirant FWO, Belgium, E-mail: Ben.Craps@fys.kuleuven.ac.be

2 E-mail: gomis@ecm.ub.es, gomis@zippy.ph.utexas.edu

${ }^{3}$ E-mail: mateos@ecm.ub.es

${ }^{4}$ Onderzoeksdirecteur FWO, Belgium, E-mail: Antoine.VanProeyen@fys.kuleuven.ac.be
} 


\section{Introduction}

Worldvolume techniques have been a powerful tool in analyzing configurations of intersecting D-branes [1, 2] (and M-branes [3]). In [1, 2] a fundamental string ending on a D-brane was analyzed from the point of view of the D-brane worldvolume theory. That theory was shown to admit solutions allowing an interpretation as fundamental strings ending on the brane. This result can be understood in terms of 'central' chargest appearing in the maximally extended worldvolume supersymmetry algebra [7]. In [8] these charges were interpreted along the lines of [9]. The Hamiltonian density was written as a sum of squares, such that putting one of the squares equal to zero yields a first order differential equation implying the equations of motion.

This worldvolume analysis was performed for branes in a flat background. The aim of the present paper is to extend the method to the case of a brane in the background produced by another brane. We have chosen the example of a D5-brane in the background of $N$ D3branes, since this configuration is physically particularly interesting. In the near horizon limit of the D3-brane geometry it is related to the baryon vertex in $\mathcal{N}=4, D=4 \mathrm{SYM}$ [10, 11]. In the non-near horizon case it is relevant for the Hanany-Witten effect [12, 13].

Two main tools are used in the analysis. First, the Hamiltonian formalism is adopted to rederive the BPS equations of [11] and [13 from a bosonic point of view. Second, this analysis is shown to fit in the description based on worldvolume superalgebras.

Section 2 contains a review of the worldvolume analysis of a D5-brane in a flat background. In Section 3 we write the Hamiltonian density as a sum of squares along the lines of [8], derive a BPS bound and interpret its physical meaning. Section 4 deals with the worldvolume superalgebra approach, showing how the charges fit in extended worldvolume algebras. The conclusions are presented in Section 5. A first appendix contains some notations. A second one gives the group theoretical structure of the worldvolume superalgebras and illustrates relations between such superalgebras.

\section{BPS Method for D-branes in a Flat background}

In this section we will review the main points of the BPS method for D-branes in a flat background [7, 8]. The relation between the BPS bounds and the charges occurring in the worldvolume supersymmetry algebra will be stressed. We will work with the particular case of a D5-brane, since in the next section we will be interested in extending this analysis to a D5-brane in a non-trivial background.

Let us thus consider a D5-brane in a flat background. The action is given by

$$
S=-T_{5} \int d^{5+1} \sigma \sqrt{-\operatorname{det}(g+F)},
$$

where $T_{5}=1 / g_{s}(2 \pi)^{5} \alpha^{\prime 3}$ is the D5-brane tension (from now onwards, we set $T_{5} \equiv 1$ ), $g$ is the induced metric and $F=d A$ is the field strength of the Born-Infeld (BI) gauge vector $A$. Let us fix the static gauge

$$
X^{0}=\sigma^{0}, \quad X^{4}=\sigma^{1}, \ldots, X^{8}=\sigma^{5}
$$

\footnotetext{
${ }^{1}$ We denote by 'central' charges the bosonic charges that appear in the right hand side of the anticommutator of the fermionic generators apart from the worldvolume coordinate transformations and $R$-symmetry generators. The terminology originates from charges allowed in 4 dimensional extended supersymmetry by [4, where these charges are really central. The anticommutator of the supersymmetries can contain analogous charges that do not commute with the Lorentz algebra and/or the $R$-symmetry [5, 6].
} 
corresponding to a brane extending (asymptotically) along directions 45678.

Now let us look for 'spike-like' static worldvolume solutions describing a fundamental string attached to the D5. We consider configurations with only one scalar excited and with purely electric BI field:

$$
X^{1}=X^{2}=X^{3}=0, \quad X^{9}=X(\sigma), \quad A_{t}=A_{t}(\sigma), \quad \vec{A} \equiv\left(A_{4}, \ldots, A_{8}\right)=\vec{A}\left(\sigma_{0}\right) .
$$

From the 10D space-time point of view one might represent the configuration we are looking for, by the following array:

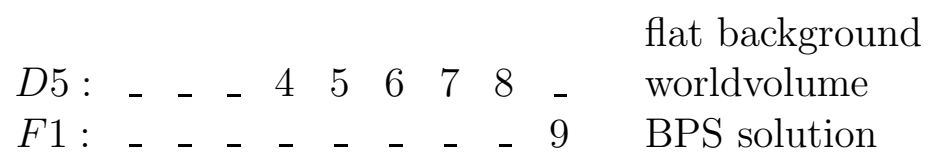

Since we are interested in bounds on the energy for these configurations, it is useful to pass to the Hamiltonian formalism. Let us denote the canonically conjugate momenta associated to $X$ and $\vec{A}$ by $P$ and $\vec{\Pi}$ respectively. The canonical phase-space Lagrangian density is defined by

$$
\mathcal{L}=\dot{\vec{A}} \cdot \vec{\Pi}+\dot{X} P-L=\vec{E} \cdot \vec{\Pi}+\dot{X} P-L-A_{t} \vec{\nabla} \cdot \vec{\Pi}
$$

where $L$ is the original Lagrangian density, $\vec{E}=\dot{\vec{A}}-\vec{\nabla} A_{t}$, and in the last step we have integrated $\vec{\nabla} A_{t} \cdot \vec{\Pi}$ by parts. In Hamiltonian form $\mathcal{L}$ is given by

$$
\mathcal{L}=\sqrt{\left(1+(\vec{\nabla} X)^{2}\right)\left(1+P^{2}\right)+\vec{\Pi}^{2}+(\vec{\Pi} \cdot \vec{\nabla} X)^{2}}-A_{t} \vec{\nabla} \cdot \Pi .
$$

The first term is the desired expression for the energy density

$$
\mathcal{H}=\sqrt{\left(1+(\vec{\nabla} X)^{2}\right)\left(1+P^{2}\right)+\vec{\Pi}^{2}+(\vec{\Pi} \cdot \vec{\nabla} X)^{2}},
$$

whereas the second term yields the Gauss law (constraint)

$$
\vec{\nabla} \cdot \vec{\Pi}=0
$$

For static configurations, $P=0$, the Hamiltonian reduces to [8]

$$
\mathcal{H}=\sqrt{(1 \pm \vec{\nabla} X \cdot \vec{\Pi})^{2}+(\vec{\nabla} X \mp \vec{\Pi})^{2}}
$$

In order to get a bound for the energy from the previous expression we should consider the 'vacuum' or ground state solution of our flat D5-brane and its energy density. The ground state solution corresponds to $X(\sigma)$ not being excited and to vanishing BI vector. This configuration is a solution of the equations of motion and its energy density is $\mathcal{E}_{g s}=1$. Hence, the energy density in (2.7) can be split as $\mathcal{H}=\mathcal{E}_{g s}+\mathcal{E}_{\text {ren }}$, where $\mathcal{E}_{\text {ren }}$ is the renormalized energy density of the brane, i.e. the energy density of the brane relative to its ground state. After this splitting, we obtain a bound for the energy density $\mathcal{E}_{\text {ren }}$ given by

$$
\mathcal{E}_{r e n} \geq|\vec{\nabla} X \cdot \vec{\Pi}|
$$

with equality when

$$
\vec{\nabla} X= \pm \vec{\Pi}
$$


The bound on the density implies the following bound on the renormalized energy:

$$
\begin{aligned}
E & =\int_{\Sigma} \mathcal{E}_{r e n} \geq\left|Z_{e l}\right|, \\
Z_{e l} & \equiv \int_{\Sigma} \vec{\Pi} \cdot \vec{\nabla} X
\end{aligned}
$$

where $\Sigma$ is the worldspace of the D5-brane.

Let us now interpret the physical meaning of $Z_{e l}$. Because of the Gauss law constraint, solutions of (2.11) correspond to solutions of $\nabla^{2} X=0$. Isolated singularities of $X$ are the BIons found in [1, 2]:

$$
X=\frac{q}{V_{(4)} r^{3}} ; \quad r^{2}=\left(\sigma^{1}\right)^{2}+\ldots+\left(\sigma^{5}\right)^{2} ; \quad V_{(4)}=\frac{8 \pi^{2}}{3}
$$

corresponding to a charge $q$ at the origin. This charge at the origin is the source of the BI vector. $[$ The Gauss law is only valid away from the singularity $r=0$, and thus

$$
Z_{e l}=q \lim _{r \rightarrow 0} X(r)
$$

where $X(r)$ represents the 'height' of the spike at a distance $r$ from the origin. The charge $q$ should be quantized in the quantum theory (see e.g. [四]). Setting it equal to its minimum value and restoring the factor $T_{5}$ we finally find

$$
Z_{e l}=T_{f} \lim _{r \rightarrow 0} X(r)
$$

where $T_{f}=1 / 2 \pi \alpha^{\prime}$ is the tension of a fundamental string. This makes clear the interpretation of $Z_{e l}$ as the energy of a fundamental string attached to the D5-brane.

Now let us show how $Z_{e l}$ appears as a central charge in the worldvolume supersymmetry algebra of the D5-brane. In order to construct this algebra it is useful to study first which bosonic background isometries preserve the vacuum solution of the D5. The worldvolume part of these symmetries is $I S O(5,1)$ and the R-symmetry part is given by $S O(4)=S U(2) \times S U(2)$. The supersymmetry generators transform in the spinor representation of this symmetry algebra, so that they can be denoted as $Q_{\alpha}^{i}$ and $\tilde{Q}_{\alpha}^{\bar{\imath}}$, where $\alpha=1 \ldots 4$ and $i, \bar{\imath}=1,2$. The 'maximally extended' superalgebra] is given by [14]:

$$
\begin{aligned}
& \left\{Q_{\alpha}^{i}, Q_{\beta}^{j}\right\}=\epsilon^{i j} \gamma_{\alpha \beta}^{\mu}\left(P_{\mu}+Z_{\mu}\right)+\gamma_{\alpha \beta}^{\mu \nu \rho} Z_{\mu \nu \rho}^{+(i j)} ; \\
& \left\{\tilde{Q}_{\alpha}^{\bar{\imath}}, \tilde{Q}_{\beta}^{\bar{j}}\right\}=\epsilon^{\bar{\imath}} \gamma_{\alpha \beta}^{\mu}\left(P_{\mu}-Z_{\mu}\right)+\gamma_{\alpha \beta}^{\mu \nu \rho} \tilde{Z}_{\mu \nu \rho}^{-(\bar{\imath})} ; \\
& \left\{Q_{\alpha}^{i}, \tilde{Q}_{\beta}^{\bar{\jmath}}\right\}=\mathcal{C}_{\alpha \beta} Y^{i \bar{\jmath}}+\gamma_{\alpha \beta}^{\mu \nu} Y_{\mu \nu}^{i \bar{\jmath}} .
\end{aligned}
$$

This algebra is the semi-direct sum of a contraction of $O S p(1 \mid 16)$ with the Lorentz and R-symmetry algebras; see Appendix B for a related discussion.

The charge $Y^{i \bar{\jmath}}$ is a vector of the R-symmetry group $S O(4)$. Interpreting this group as the rotation group of the four-dimensional space transverse to the D5 [7], we see that $Y^{i \bar{\jmath}}$ selects a direction in this transverse space, as a string ending on the D5 does. Hence the

\footnotetext{
${ }^{2}$ The initial generic configuration had no source for the BI vector. The case of a D5 in the background of a D3 studied in the next section will be different since for a generic configuration there is a source for the $\mathrm{BI}$ vector due to the Wess-Zumino term.

${ }^{3}$ In this algebra one allows the most general anticommutator of the supersymmetries. This corresponds to extending the original symmetry algebra of the D5-brane, which we call the 'worldvolume algebra', to a larger one encoding all BPS intersections.
} 
charge $Y^{i \bar{\jmath}}$ is associated to fundamental strings ending on the D5-brane. Its expression in terms of the fields of the D5-brane ist

$$
Y^{M}=\int_{\Sigma} \vec{\Pi} \cdot \vec{\nabla} X^{M}
$$

where $X^{M}$ is a vector in the transverse space to the D5 brane, i.e. along the directions 1239. Thus we recognize $Z_{e l}$ as the central charge $Y^{i \bar{\jmath}}$ appearing in the worldvolume supersymmetry algebra (in our expression (2.12) the direction $M=9$ has been chosen out of four equivalent ones). The other charges occurring in the algebra are also interpreted as intersections of branes along the lines of [7, 14].

\section{The D5 in the D3 background: Hamiltonian analysis}

In this section we will derive a BPS bound on the energy of a D5-brane in the background geometry of a stack of $N$ D3-branes, by showing that it is bounded from below by a topological quantity. Let us thus begin by describing the D3 background. The ten-dimensional metric is

$$
d s_{(10)}^{2}=H^{-1 / 2} d X_{\|}^{2}+H^{1 / 2}\left(d r^{2}+r^{2} d \Omega_{(5)}^{2}\right) .
$$

Here $X_{\|}=\left(X^{0}, X^{1}, X^{2}, X^{3}\right)$ are cartesian coordinates on $\mathbb{E}^{(1,3)}$ and $d \Omega_{(5)}^{2}$ is the line element on a unit five-sphere, which we take to be parametrized by standard angular coordinates $\Theta, \Phi^{i}, i=1, \ldots, 4$, where $\Phi^{i}$ are coordinates on a four-sphere. Thus we have

$$
d \Omega_{(5)}^{2}=d \Theta^{2}+\sin ^{2} \Theta d \Omega_{(4)}^{2}
$$

The coordinate $r$ parametrizes the radial distance to the branes, so $r, \Theta$, and $\Phi^{i}$ are spherical coordinates on the six-dimensional space transverse to the branes. The harmonic function appearing above is

$$
H=a+\frac{R^{4}}{r^{4}}
$$

Here $a=0$ describes the near-horizon geometry $A d S_{5} \times S^{5}$, whereas $a=1$ describes an asymptotically flat space-time. The parameter $R$ measures the gravitational size of the stack of branes, and in the case $a=0$ precisely coincides with the radius of both $A d S_{5}$ and $S^{5}$. On top of the metric, the supergravity solution describing the D3-branes is characterized by a non-vanishing Ramond-Ramond (RR) 5-form field strength

$$
\begin{aligned}
& G^{(5)}=-H^{-2} H^{\prime} d X^{0} \wedge d X^{1} \wedge d X^{2} \wedge d X^{3} \wedge d r+4 R^{4} \omega_{(5)} \\
& \omega_{(5)}=\sin ^{4} \Theta d \Theta \wedge \omega_{(4)},
\end{aligned}
$$

where $\omega_{(n)}$ is the volume form on a unit $n$-sphere and $H^{\prime} \equiv \partial_{r} H$. Now let us consider a D5-brane coupled to the above background. It will be described by the action

$$
S=-T_{5} \int_{\Sigma} d^{6} \sigma \sqrt{-\operatorname{det}(g+F)}+T_{5} \int_{\Sigma} A \wedge G^{(5)} .
$$

Note that the RR field acts as source for the worldvolume gauge field $A$ through its coupling in the Wess-Zumino term above. For all the configurations we will consider, only the second term in (3.4) will contribute to this coupling. The inclusion of this term is therefore essential for the correct treatment of such configurations.

\footnotetext{
${ }^{4}$ This expression should be obtainable from the explicit form of the supercharges, which one could get via Noether's theorem as in [15].
} 
We are interested in passing to the Hamiltonian formalism in order to derive a bound on the energy. Let us first of all fix the static gauge by choosing $\sigma=\left\{\theta, \varphi^{i} ; i=1, \ldots, 4\right\}$ as coordinates on the D5-brane worldvolume and by identifying

$$
X^{0}=t, \quad \Theta=\theta, \quad \Phi^{i}=\varphi^{i} .
$$

Since we will only be interested in configurations which preserve the $S O(5)$ symmetry which rotates the $\Phi^{i}=\varphi^{i}$ coordinates, we also restrict ourselves to the following type of configurations:

$$
X^{1}=X^{2}=X^{3}=0, \quad r=r(t, \theta), \quad A_{t}=A_{t}(t, \theta), \quad A_{\theta}=A_{\theta}(t, \theta), \quad A_{\varphi^{i}}=0 .
$$

Under these conditions, the D5 action reduces to

$$
S=T_{5} V_{(4)} \int d t d \theta \sin ^{4} \theta\left[-H r^{4} \sqrt{r^{2}+r^{\prime 2}-H \dot{r}^{2} r^{2}-E^{2}}+4 R^{4} A_{t}\right],
$$

where $\dot{r} \equiv \partial_{t} r, r^{\prime} \equiv \partial_{\theta} r, E \equiv F_{0 \theta}$ and $V_{(4)}=8 \pi^{2} / 3$ is the volume of a unit four-sphere. From now on we will set $T_{5} V_{(4)} \equiv 1$. The canonical momenta $P$ and $\Pi$ conjugate to the fields $r$ and $A_{\theta}$ in the above action are

$$
\begin{aligned}
P & \equiv \frac{\partial L}{\partial \dot{r}}=\sin ^{4} \theta \frac{H^{2} r^{6} \dot{r}}{\sqrt{r^{2}+r^{\prime 2}-H \dot{r}^{2} r^{2}-E^{2}}} \\
\Pi & \equiv \frac{\partial L}{\partial \dot{A}_{\theta}}=\frac{\partial L}{\partial E}=\sin ^{4} \theta \frac{H r^{4} E}{\sqrt{r^{2}+r^{\prime 2}-H \dot{r}^{2} r^{2}-E^{2}}} .
\end{aligned}
$$

The canonical phase-space Lagrangian density is defined by

$$
\mathcal{L}=\dot{A_{\theta}} \Pi+\dot{r} P-L=E \Pi+\dot{r} P-L-A_{t} \Pi^{\prime},
$$

where in the last step we have integrated $A_{t}^{\prime} \Pi$ by parts. Inverting the relations (3.10) one can rewrite $\mathcal{L}$ in the Hamiltonian form

$$
\mathcal{L}=\sqrt{\left(r^{2}+r^{\prime 2}\right)\left(\frac{P^{2}}{H r^{2}}+\Pi^{2}+\Delta^{2}\right)}+A_{t}\left(-\Pi^{\prime}-4 R^{4} \sin ^{4} \theta\right),
$$

where

$$
\Delta \equiv H r^{4} \sin ^{4} \theta
$$

The first term is just the desired energy density

$$
\mathcal{H}=\sqrt{\left(r^{2}+r^{\prime 2}\right)\left(\frac{P^{2}}{H r^{2}}+\Pi^{2}+\Delta^{2}\right)}
$$

whereas the second term yields the 'modified' Gauss law $\partial_{\theta} \Pi=-4 R^{4} \sin ^{4} \theta$. Remark that in the flat background case we had as Gauss law $\vec{\nabla} \cdot \vec{\Pi}=0$, while here we have a source term, a direct consequence of the Wess-Zumino coupling to $G^{(5)}$ in the D5-brane action. This is the difference referred to in footnote 2.

The Gauss law constraint is solved by 13.

$$
\Pi(\nu, \theta)=\frac{1}{2} R^{4}\left[3(\nu \pi-\theta)+3 \sin \theta \cos \theta+2 \sin ^{3} \theta \cos \theta\right],
$$


where $\nu$ is an integration parameter. Its meaning will become clear below (see also [13]). We thus use the solution of the Gauss law constraint, but do not use the field equation for $r(\theta)$, which will rather be implied by BPS conditions.

We further consider static configurations. The energy density reduces to

$$
\mathcal{H}=\sqrt{\left(r^{2}+r^{2}\right)\left(\Pi^{2}+\Delta^{2}\right)} .
$$

The first key observation to prove the existence of a BPS bound on the energy of the D5 is that the above expression can be rewritten as

$$
\mathcal{H}=\sqrt{\mathcal{Z}_{e l}^{2}+r^{2}(\Delta \cos \theta-\Pi \sin \theta)^{2}\left(\frac{r^{\prime}}{r}-f\right)^{2}}
$$

where

$$
\begin{aligned}
\mathcal{Z}_{e l} & \equiv r(\Delta \cos \theta-\Pi \sin \theta)\left(1+\frac{r^{\prime}}{r} f\right), \\
f(a, \nu ; r, \theta) & \equiv \frac{\Delta(a, r, \theta) \sin \theta+\Pi(\nu, \theta) \cos \theta}{\Delta(a, r, \theta) \cos \theta-\Pi(\nu, \theta) \sin \theta} .
\end{aligned}
$$

The function $r(\theta)$ has in general a limited range in $\theta$ from $\theta_{i}$ to $\theta_{f}$. The second key observation is that the integral

$$
Z_{e l} \equiv \int_{\theta_{i}}^{\theta_{f}} d \theta \mathcal{Z}_{e l}
$$

is topological, as we will show below. This implies the desired BPS bound on the D5 energy, namely

$$
E_{\text {ren }}+E_{g s} \equiv \int_{\theta_{i}}^{\theta_{f}} d \theta \mathcal{H} \geq\left|Z_{e l}\right|
$$

through the following two inequalities

$$
E_{\text {ren }}+E_{g s}=\int_{\theta_{i}}^{\theta_{f}} d \theta \mathcal{H} \geq \int_{\theta_{i}}^{\theta_{f}} d \theta\left|\mathcal{Z}_{e l}\right| \geq\left|Z_{e l}\right|
$$

Above, $E_{g s}$ is the ground state or vacuum energy of the D5-brane in the D3 background, and $E_{r e n}$ is the renormalized energy of a configuration, i.e. the energy relative to this ground state. Of course, $E_{g s}$ might be a divergent quantity; only energies relative to it are physical. We will come back to this issue below.

The BPS bound (3.21) is saturated if and only if both inequalities above are. Saturation of the first onf yields a first order differential equation on the D5 embedding $r(\theta)$, namely

$$
\frac{r^{\prime}}{r}=f
$$

This equation is valid for both the near-horizon case and the asymptotically flat one (note that the function $f$ is different for the two cases). In the former case, it was derived in [11] by imposing the preservation of some fraction of global worldvolume supersymmetry. For the latter case, it was proposed in [13 as a plausible generalization of the result in 11 and shown to imply the equations of motion of the D5. In our approach, both cases can be dealt with at once, and in both of them the BPS equation emerges from a bound on the D5 energy.

\footnotetext{
${ }^{5}$ If $r(\Delta \cos \theta-\Pi \sin \theta)=0$, the introduction of $f$ in $(3.17)$ is not allowed. In this case, saturation of the BPS bound implies that the numerator of $f$ is zero too, leading to $\Pi(\nu, \theta)=\Delta(a, r, \theta)=0$, which implies $\theta=0$ or $\theta=\pi$, and can thus be neglected.
} 
On top of (3.23), we still have to impose saturation of the second inequality in (3.22) to saturate the energy bound (3.21). Such inequality is saturated when $\mathcal{Z}_{e l}$ does not change sign in the integration region $\left[\theta_{i}, \theta_{f}\right]$. This condition determines the possible values of $\theta_{i}$ and $\theta_{f}$ (for BPS solutions), where boundary conditions for $r$ should be supplemented. When (3.23) holds, the sign of $\mathcal{Z}_{\text {el }}$ is determined by the sign of

$$
\Delta \cos \theta-\Pi \sin \theta=a r^{4} \sin ^{4} \theta \cos \theta+\frac{3}{2} R^{4} \sin \theta \eta(\theta),
$$

where the function

$$
\eta(\theta) \equiv \theta-\pi \nu-\sin \theta \cos \theta
$$

is monotonically increasing for $\theta \in[0, \pi]$. In $[13$ the restriction was made to $\nu \in[0,1]$. We make the same restriction in the main text. In this case, $\eta$ changes sign precisely once in this interval at $\theta_{0}$ such that $\eta\left(\theta_{0}\right)=0$. Therefore, in the case $a=0$, the second inequality in (3.22) is saturated if

$$
\theta_{i}=0, \quad \theta_{f}=\theta_{0}
$$

or

$$
\theta_{i}=\theta_{0}, \quad \theta_{f}=\pi
$$

These two possibilities coincide with those found in [13], where they were determined by explicitly solving the BPS equation (3.23).

Although the procedure is conceptually the same, in the case $a=1$ we cannot determine from this analysis the possible range of $\theta$. To determine the sign of (3.24) we would need the explicit form of the solution $r(\theta)$. These solutions were studied in [13] only numerically (in the case $a=1$ ): no analytic solution was found.

Let us come back to the topological nature of $Z_{e l}$. Since the density $\mathcal{Z}_{e l}$ can be rewritten as a total derivative,

$$
r(\Delta \cos \theta-\Pi \sin \theta)\left(1+\frac{r^{\prime}}{r} f\right)=\frac{d}{d \theta}\left\{\Pi r \cos \theta+\left(\frac{a}{5}+\frac{R^{4}}{r^{4}}\right)(r \sin \theta)^{5}\right\},
$$

it follows that

$$
Z_{e l}=[\Pi r \cos \theta]_{\theta_{i}}^{\theta_{f}}+\left[\left(\frac{a}{5}+\frac{R^{4}}{r^{4}}\right)(r \sin \theta)^{5}\right]_{\theta_{i}}^{\theta_{f}}
$$

depends only on the boundary conditions $r\left(\theta_{i}\right)$ and $r\left(\theta_{f}\right)$. This means that, for fixed values of $r\left(\theta_{i}\right)$ and $r\left(\theta_{f}\right), Z_{e l}$ is invariant under local variations of the fields. It is in this sense that it is a topological quantity. Configurations saturating the bound (3.21) minimize the energy for fixed boundary conditions, and therefore they automatically solve the equations of motion.

For $a=1$, the second term is precisely the ground state energy of the D5. Indeed, consider an infinite planar D5-brane intersecting the stack of D3-branes at a point

$$
\begin{array}{llllllllllll}
D 3: & 1 & 2 & 3 & - & - & - & - & - & - & \text { background } \\
D 5: & - & - & - & 4 & 5 & 6 & 7 & 8 & - & \text { worldvolume. }
\end{array}
$$

\footnotetext{
${ }^{6}$ Recall that $0 \leq \theta_{i} \leq \theta_{f} \leq \pi$ since we identified $\theta$ with $\Theta$, one of the angular coordinates on the $S^{5}$.

${ }^{7}$ If one were to consider solutions with $\nu$ outside this range, $\eta$ would have a definite sign, so that, for $a=0$, the solution $r(\theta)$ can extend over the whole interval $[0, \pi]$, developing spikes both at $\theta=0$ and $\theta=\pi$.

${ }^{8}$ Only the Gauss law solution (3.15) has to be used to deduce (3.28). Neither the field equation for $r(\theta)$ nor the BPS condition (3.23) are needed.
} 
The D5 worldspace $\Sigma$ spans a five-dimensional hyperplane in the transverse space to the D3branes, which we can take as the $\theta=\pi / 2$ hyperplane. The energy of such a configuration is

$$
E_{g s}=\int_{\Sigma} d^{5} \sigma \sqrt{-\operatorname{det} g}=\int_{0}^{r_{f}} d r r^{4} H(r)=\left[\left(\frac{a}{5}+\frac{R^{4}}{r^{4}}\right) r^{5}\right]_{0}^{r_{f}},
$$

which can be seen to agree with the second term in (3.29) by making use of the form of the solutions found in [13].

In the case $a=0$, the second term in (3.29) vanishes for all the solutions of (3.23) (again, see [13]). We will see below that the first term in (3.29) has a clear physical interpretation not associated with any ground state energy, so we conclude that the D5 ground state for $a=0$ is such that its energy vanishes. We interpret this fact in the following way. Let us understand the ground state of the D5 in the near horizon geometry of the D3-branes as a limit of the D5 ground state in the full D3 geometry. The latter is represented by the array (3.30). One can think of it as an intersection of orthogonal D5 and D3 branes in a point, but this is not necessarily the case: since it is a BPS configuration, both kinds of branes can be separated along the 9th direction while preserving the BPS character. Hence, generically, in the full D3 geometry, the D5 in its ground state does not intersect the D3-branes. Taking the near horizon limit means zooming up the region close to the latter. If the D5 was placed at a certain finite distance along $X^{9}$, then it will 'disappear to infinity' when one focuses on regions arbitrarily close to the D3-branes. In this sense, its ground state energy in the full geometry will not show up after taking the near horizon limit.

The D5 ground state we end up with taking this limit can be identified with the $\nu=1$ configuration found in [13]. The reason is that configurations with $\nu \neq 1$ in [13 represent a D5-brane infinitely far away from the D3-branes, connected to them by $(1-\nu) N$ strings. The limit $\nu \rightarrow 1$ can thus be interpreted as the limit in which only the D5 in its ground state 'at infinity' remains. This remark is important because although in the strict limit the D5 disappears, in taking such a limit there is always a selected direction, namely $X^{9}$. This will be essential in the next section.

Having identified $E_{g s}$ for both the $a=0$ and the $a=1$ cases, we can rewrite the bound (3.21) as

$$
E_{\text {ren }} \geq\left|Z_{9}\right|, \quad Z_{9} \equiv[\Pi r \cos \theta]_{\theta_{i}}^{\theta_{f}}
$$

The physical meaning of $Z_{9}$ can (again) be understood by evaluating it for the solutions of (3.23). Restoring the factor $T_{5} V_{(4)}$ the result is

$$
Z_{9}=n T_{f} L, \quad n=\left\{\begin{array}{cc}
(1-\nu) N & \text { for } a=0 \\
\left(\frac{1}{2}-\nu\right) N & \text { for } a=1
\end{array}\right.
$$

where $L \equiv r\left(\theta_{f}\right)$ for $a=0$ and $L \equiv r\left(\theta_{f}\right) \cos \left(\theta_{f}\right)$ for $a=1$. The above expression clarifies the physical interpretation of $Z_{9}$. It is the energy of $n$ fundamental strings of length $L$ and tension $T_{f}$ joining together the D5 and the D3-branes. From the ten-dimensional space-time point of view this corresponds to the triple intersection

$$
\begin{array}{lllllllllll}
D 3: & 1 & 2 & 3 & - & - & - & - & - & - & \text { background } \\
D 5: & - & - & - & 4 & 5 & 6 & 7 & 8 & - & \text { worldvolume } \\
F 1: & - & - & - & - & - & - & - & - & 9 & \text { BPS solution. }
\end{array}
$$

In our analysis, the D3 is the background and the F1 plays the role of a soliton of the D5 worldvolume theory.ण

\footnotetext{
${ }^{9}$ In the case $a=0, \nu=1$ (see [13]) there is an additional contribution to $Z_{9}$ given by $-\Pi\left(\theta_{i}\right) r\left(\theta_{i}\right)$. We neglect it because it is finite, whereas $Z_{9}$ is divergent. It is precisely the form of this divergence that we would like to interpret.

${ }^{10}$ Triple intersection from the point of view of worldvolume theories have been studied in [7], see also [16].
} 
We have chosen the name $Z_{9}$ because of the direction in which the string stretches. In the case $a=0$, the D5 is infinitely far away from the D3-branes, so $L$ is divergent. In the case $a=1$, the D5 is asymptotically a flat hyperplane and $L$ coincides with its distance to the point $r=0$, where the D3-branes are.

The solutions of the BPS equation (3.23) are thus the D5 worldvolume description (spikelike solutions) of the configuration (3.34). In the next section we will provide further evidence in favor of the interpretation of $Z_{9}$ as a charge associated to the ending of fundamental strings on the D5 worldvolume from its appearance as a central charge in the D5 worldvolume supersymmetry algebra.

\section{Algebras}

In the previous section we have understood the BPS equation (3.23) from a bosonic point of view. Now we will show that the charge $Z_{9}$, which sets the bound (3.32), is a central charge in the worldvolume supersymmetry algebra of the D5 in the D3 background. To do so, we will determine this algebra. The cases $a=0$ and $a=1$ will be treated separately, since the results are different. However, the procedure to determine the algebra is conceptually the same, and in principle applies to any brane in any curved background.

The starting point is to identify the ground state configuration of the brane in the given background. Two remarks are in order here. First, one should choose it according to which excited configurations one would like to study. From a space-time point of view, this corresponds to which type of triple intersections one would like to consider. For instance, suppose instead of (3.34) we had been interested in studying the intersection

$$
\begin{array}{lllllllllll}
D 3: & 1 & 2 & 3 & - & - & - & - & - & - & \text { background } \\
D 5: & - & 2 & 3 & 4 & 5 & 6 & - & - & - & \text { worldvolume } \\
D 3: & 1 & - & - & 4 & 5 & - & - & - & - & \text { BPS solution. }
\end{array}
$$

Then the vacuum configuration would have consisted of an static infinite planar D5-brane intersecting the stack of D3-branes in a 2-brane. The BPS-solution would have been described by the scalar $X^{1}$ and an excited BI gauge field.

Second, the starting point is purely bosonic, since one need only consider a bosonic background.

Next, one determines which isometries of the background preserve the ground state configuration; these isometries give rise to linearly realized symmetries of the brane worldvolume theory [17]: they constitute the worldvolume bosonic symmetry algebra. Part of them are associated to worldvolume coordinate transformations and part of them to R-symmetry.

To determine the whole worldvolume supersymmetry algebra, one first imposes that the supersymmetry generators are in the (fundamental) spinorial representation of the algebra of worldvolume coordinate transformations. Moreover, they should be in a representation of the R-symmetry algebra. That is usually sufficient to fix the number of supercharges of the worldvolume supersymmetry algebra. In the case $a=0$ one can make use of the classification of Nahm [18] of super-adS or superconformal algebras

To definitely fix this algebra, only the anticommutator of these supercharges needs still to be determined. This is done by allowing all the original bosonic generators not forbidden by Jacobi identities to occur.

\footnotetext{
${ }^{11}$ In principle, the supersymmetry generators are determined from the Killing spinors of the abovementioned bosonic ground state configuration. One could have started from the whole isometry superalgebra of the (super)background and have determined which of these superisometries preserve the ground state of the brane.
} 
The algebra constructed this way, is what we call the worldvolume supersymmetry algebra. The only bosonic generators it contains, are those originating from bosonic isometries of the background.

The final step in the procedure is to enlarge this algebra along the lines of [7] to what we will call the maximally extended worldvolume supersymmetry algebra. This is done by constructing the most general anticommutator of the supersymmetry generators allowed by symmetry by adding all possible central extensions (see footnote 1). The algebra obtained in this way is the one which encodes all the BPS intersections. Such intersections involve the brane whose worldvolume theory we are considering, the branes creating the background and a third one. The latter is the one associated to the central charges we have enlarged the worldvolume algebra with.

Note that this procedure does not completely fix the superalgebra yet, because so far one has not determined the commutators of the central charges with the supersymmetry generators, with the original bosonic generators and with themselves! An explicit computation of the charges via Noether theorem would allow one to fix this ambiguity. However, consistency with Jacobi identities fixes in many cases this algebra, as we will see below.

In the rest of this section we will illustrate the above general procedure by applying it to a D5 brane in a D3 background. We will show the appearance of $Z_{9}$, and also interpret the central charges in the supersymmetry algebra.

\subsection{The near-horizon algebra}

The first task is to determine which bosonic isometries of the background preserve the ground state of the D5-brane. Recall that the near-horizon D3 background has as worldvolume superalgebra $S U(2,2 \mid 4)$, with bosonic isometry group $S O(4,2) \times S O(6)$. As already discussed in section 3, when the D5 is in its ground state its worldspace consists of a hyperplane extending along directions 45678, located at arbitrary constant values of $X^{i}, i=1,2,3$, for instance $X^{i}=0$, and at $X^{9} \rightarrow \infty$.

This configuration is preserved by the subgroup $S O(2,1) \times S O(5) \times S O(3)$ of the background bosonic isometries. Indeed, the condition $X^{1}=X^{2}=X^{3}=0$ is preserved by an $S O(2,1) \times S O(3)$ subgroup of the $S O(4,2)$ factor, and selecting the directions 45678 (or alternatively, the 9 th) breaks the $S O(6)$ factor to $S O(5)$.

The factors of the symmetry group have the following interpretation: the $S O(2,1)$ con-

tains time translations, dilations and boosts for the radial coordinate $r=\sqrt{\sum_{i=4}^{9}\left(X^{i}\right)^{2}}$. The $S O(5)$ contains the D5 worldspace rotations and the $S O(3)$ is the group of D3 worldspace rotations, which is, from the point of view of the D5 brane supersymmetry group, the Rsymmetry.

We have thus identified the bosonic worldvolume symmetry group. The 32 fermionic generators of the D3 worldvolume algebra are in the representation $(4, \overline{4})+(\overline{4}, 4)$ of $S O(4,2) \oplus$ $S O(6)$. The intersection with $D 5$ preserves half of them, which are still spinors of $S O(2,1)$, $S O(5)$ as well as $S O(3)$. They are thus in the $(2,4,2)$ representation of the bosonic worldvolume algebra. Using the classification of Nahm [18] (for the terminology we use, see the review in [19]) we can identify therefore the worldvolume superalgebra as $O S p\left(4^{*} \mid 4\right)$, using the identification $S O^{*}(4)=S O(2,1) \times S O(3)$, and $U S p(4)=S O(5)$. The superalgebra is in this way completely fixed. On the worldvolume half of the supersymmetries are ordinary $Q$-supersymmetries, and the other half are $S$-supersymmetries, as distinguished by the eigenvalue of the dilatations. This conformal worldvolume superalgebra originates from the super-adS symmetry in spacetime [17, 19]. In the latter form the fermionic generators $Q$ and $S$ are unified in a supersymmetry $Q$. In this formulation the anticommutators of these 
supersymmetries are

$$
\left\{Q_{\alpha}^{i}, Q_{\beta}^{j}\right\}=C_{\alpha \beta} R^{[i j]}-\frac{1}{8} \gamma_{\alpha \beta}^{\mu \nu} M_{[\mu \nu]} \delta^{i j}
$$

where $\alpha, \beta=1, \ldots 4$ and $\mu, \nu=4, \ldots 8$ are spinor and vector indices of $S O(5)$, respectively. The $i, j=1, \ldots 4$ are vector indices of $S O^{*}(4)$. At the right hand side appear $R^{[i j]}$, the generators of $S O^{*}(4)$, and $M_{[\mu \nu]}$, the generators of $S O(5)$. In the conformal notation of this algebra, the latter appear only in the $\{Q, S\}$ anticommutator. The commutators of the bosonic algebra and the supersymmetries just reflect that the supersymmetries are in the $(2,4,2)$ representation.

Having identified the worldvolume algebra as $O S p\left(4^{*} \mid 4\right)$, we now look for the extended worldvolume algebra. In the spirit of [7] we thus enlarge this algebra by all possible generators that can appear on the right hand side

$$
\left\{Q_{\alpha}^{i}, Q_{\beta}^{j}\right\}=C_{\alpha \beta} R^{[i j]}-\frac{1}{8} \gamma_{\alpha \beta}^{\mu \nu} M_{[\mu \nu]} \delta^{i j}+\gamma_{\alpha \beta}^{\mu} U_{\mu}^{[i j]}+\gamma_{\alpha \beta}^{\mu \nu} Z_{[\mu \nu]}^{(i j)_{s}},
$$

where $(i j)_{s}$ indicates that $Z$ is symmetric traceless. The Jacobi identities do not allow that the generators $U$ and $Z$ commute with the supersymmetries. Indeed this is clear e.g. from the Jacobi identity $[Q, Q, Z]$, due to the fact that $S O^{*}(4)$ and $S O(5)$, which rotate the supersymmetries, appear in the right hand side of (4.3). All the bosonic generators should thus be part of this simple Lie superalgebra which contains the conformal algebra. Looking through the list of all superalgebras [20] (see [19] for a convenient table), one finds that the extended worldvolume superalgebra is $O S p(1 \mid 16)$. The right hand side of (4.3) is a symmetric $16 \times 16$ matrix $M_{A B}$ where $A=(\alpha i)$. Introducing the symplectic metric $\Omega^{A B}=\mathcal{C}^{\alpha \beta} \delta^{i j}$, one obtains the algebra

$$
\begin{aligned}
& \left\{Q_{A}, Q_{B}\right\}=M_{A B}, \quad\left[M_{A B}, Q_{C}\right]=Q_{(A} \Omega_{B) C} \\
& {\left[M_{A B}, M_{C D}\right]=\Omega_{A(C} M_{D) B}+\Omega_{B(C} M_{D) A},}
\end{aligned}
$$

which thus defines the full algebra. The $S p(16)$ generators are decomposed in (4.3) under $S O(2,1) \times S O(5) \times S O(3)$ as

$$
[(3,1,1)+(1,1,3)]+(1,10,1)+[(3,5,1)+(1,5,3)]+(3,10,3) .
$$

For the splitting of fermionic generators in $Q$ and $S$ supersymmetries, we have to write the $S O^{*}(4)$ vector index $i$ as $(\kappa \pm)$, where $\kappa=1,2$ is a spinor index of $S O(3)$ and \pm indicates the $S O(2,1)=S U(1,1)$ index. The ordinary $(Q)$ supersymmetries are those where this latter index is + . The anticommutator of these ordinary supersymmetries is then obtained from (4.3) as

$$
\left\{Q_{\alpha}^{\kappa}, Q_{\beta}^{\lambda}\right\}=C_{\alpha \beta} \tilde{R} \epsilon^{\kappa \lambda}+\gamma_{\alpha \beta}^{\mu} \tilde{U}_{\mu} \epsilon^{\kappa \lambda}+\gamma_{\alpha \beta}^{\mu \nu} \tilde{Z}_{[\mu \nu]}^{(\kappa \lambda)},
$$

where $\tilde{R}, \tilde{U}$ and $\tilde{Z}$ are the $(++)$ parts of $R, U$ and $Z$.

The charge $Z_{9}$ is a component of a 3 -vector of $S O(2,1)$. Indeed, it is proportional to the value of $r$ at the singularity, see (3.33), and $\left(r, r t,-r t^{2}+R^{2} / r\right)$ is a 3 -vector, the one used to embed $A d S_{2}$ in a 3-dimensional flat space (see e.g. (17) in [19] with $z=r^{-1}$ and $x^{\mu}=t$ ). Under the remaining factors of the bosonic symmetry group it is invariant, and thus we conclude that $Z_{9}$ is part of a $(3,1,1)$. It can thus be combined with $(E, D, K)$, the generators of energy, dilatations and Lorentz boosts in $(t, r)$, to appear in $R$. The $(3,1,1)$ part of the generators $R^{[i j]}$ in $(4.3)$ is the triplet of $S O(2,1)$ whose $(++)$ component is ${ }^{[2]}$

$$
\tilde{R}=\left(E-Z_{9}\right) \text {. }
$$

\footnotetext{
${ }^{12}$ In the IIB susy algebra in 10 dimensions, the central charge $Z_{9}$ and the energy $E$ occur separated. The breakdown of this algebra to the $Q$ supersymmetry part of the extended worldvolume algebra (4.6) should lead to this combination.
} 
(As before, for the definition of $E$ we have put the energy of the vacuum configuration of the D5 equal to zero.) The fact that this combination $E-Z_{9}$ appears on the right-hand side of the anticommutator of the supersymmetry generators implies that a configuration with non-vanishing string charge along the 9-direction (and the other central charges equal to zero) preserves all 16 supersymmetries if the energy is chosen to saturate the corresponding BPS-bound.

Indeed, from a space-time point of view it is easy to see that, having performed the projections onto supercharges preserving the D3-D5 intersecting (or overlapping) on a point, one can put an additional string in the remaining direction 'for free' (see, for instance, [13]).

To summarize the main point of this subsection, we have shown that $Z_{9}$ has indeed the right quantum numbers to be interpreted as a central charge in the worldvolume supersymmetry algebra of the D5-brane in the near-horizon limit of the D3 background; and that doing so makes clear that the corresponding BPS-solutions (the strings in the 9th-direction) preserve all 16 supersymmetries of this algebra.

\subsection{The non-near-horizon case}

We now move on to discuss the $a=1$ case. The background bosonic isometry group is $I S O(3,1) \times S O(6)$. The vacuum configuration consists of a hyperplane extending along directions 45678, located at arbitrary values of $X^{i}, i=1,2,3$ and $X^{9}$. Therefore, the worldvolume bosonic symmetry group is $\mathbb{R} \times S O(5) \times S O(3)$. Both the worldvolume superalgebra and its maximal extension are obtained by applying the general procedure, as was done in the near-horizon case. However, it can also be obtained from the $a=0$ results. We find this alternative illustrative, so we describe it here.

Comparing to the $a=0$ case, we see that the $S O(2,1)$ conformal symmetry appearing there, has been broken to just time translations $\mathbb{R}$, its Poincaré part. In general, the Poincaré algebra is not a simple algebra, it is the semi-direct sum of translations and the Lorentz algebra. In this case, the latter is trivial, because there is just the time direction [3].

Similarly, the worldvolume superalgebra $O S p\left(4^{*} \mid 4\right)$ from the $a=0$ case is broken, for $a=$ 1 , to an algebra which is the super-Poincaré subalgebra of the 1-dimensional superconformal algebra四. The latter contains as first part the $Q$-supersymmetries and what appears in their anticommutator, i.e. the (time) translations. This part satisfies Jacobi identities by itself. But it is not a simple superalgebra. The full super-Poincaré algebra is the semi-direct sum of this first part with the Lorentz algebra, and the automorphism group of the supersymmetries. The conformal algebra is here $S O(2,1)$, and has no Lorentz subalgebra. The automorphism group is $S O(3) \oplus S O(5)$. The $S O(5)$ rotations belong to the worldvolume algebra for the D5, being its worldspace rotations. For D5, $S O(3)$ is the R-symmetry. This is the worldvolume algebra for $a=1$.

The extended worldvolume superalgebra can again be obtained from the one in the near-horizon case using the breakdown of the conformal algebra. With the breaking of the conformal algebra, also its representations are cut. The $Q$ and $S$ supersymmetries together form a representation of the conformal algebra, here $S O(2,1)$. The supersymmetries are distinguished according to their eigenvalue under the dilatations, which is, with suitable normalization, $\pm \frac{1}{2}$. The ones with positive weight, i.e. the $Q$ supersymmetries, are present,

\footnotetext{
${ }^{13}$ Note that not the full worldvolume coordinate transformation algebra is conformal for $a=0$, but only the part corresponding to the intersection point, which is the 1-dimensional conformal algebra $S O(2,1)$. The worldvolume coordinate transformations contain also the $S O(5)$ rotations, which are from the point of view of the superconformal theory of the point, an R-symmetry.

${ }^{14}$ This super-Poincaré algebra can alternatively be seen as a contraction of the super-adS algebra. We come back to these issues in appendix B.
} 
while the ones with negative eigenvalue, the $S$ supersymmetries, are not any more present. Similarly the central charges $U$ and $Z$ in (4.3) can be split according to their eigenvalue under the dilatation operator, which is 1,0 or -1 . Only the former appear in the anticommutator of two $Q$ supersymmetries, and they thus remain in the super-Poincaré algebra, while the others do not. Those which remain are the $\tilde{U}$ and $\tilde{Z}$ as in (4.6). The bosonic generators in there mutually commute and commute with the supersymmetries. The full extended worldvolume algebra thus consists of these generators and in general the Lorentz generators (not present in this case) and the R-symmetry generators of the superconformal algebra, which are here the $S O(3)$ part of the $S O^{*}(4)$ generators $R^{[i j]}$ as R-symmetries for the D5 brane, and $M_{\mu \nu}$, the generators of $S O(5)$ worldspace rotations.

Still a third possibility to obtain the full extended worldvolume superalgebra consists in starting from the one of a D5-brane in a flat background [14] and restricting to the generators preserved when a D3-brane is added to the configuration.

The result can be described as a contraction of $O S p(1 \mid 8)$. The latter contains the anticommutator (4.6), but with bosonic operators that do not commute mutually or with the supersymmetries, similar to (4.4). The contraction consists in scaling the fermionic generators with $x$, the bosonic ones with $x^{2}$, and then taking the limit $x \rightarrow \infty$.

As in the $a=0$ case we have the identification (4.7). Note that now $Z_{9}$ is a singlet under the whole symmetry group, as corresponds to the fact that now, in contrast to the $a=0$ case, this group does not act on the coordinate $r$ to which $Z_{9}$ is proportional. Apart from this difference, the conclusions about $Z_{9}$ are the same as in the $a=0$ case: it has the interpretation of a fundamental string in the 9 th direction additional supersymmetries if the energy is properly chosen.

\subsection{Interpretation of the central charges}

Let us start with the $a=1$ case. In addition to the combination (4.7) of energy minus string charge, the algebra (4.6) contains the charges $\tilde{U}_{\mu}$ and $\tilde{Z}_{[\mu \nu]}^{(\kappa \lambda)}$. Using standard arguments (see for instance 21] for examples in the M-theory context), one can show that these correspond to $1 / 2$ BPS solutions of the worldvolume theory. Bearing in mind the interpretation of the R-symmetry as rotations in the D3-brane worldspace, these charges suggest a spacetime interpretation of the corresponding worldvolume solutions [7]. In our case they can be interpreted as a third brane intersecting the D5-brane in the D3 background, preserving 1/8 supersymmetry (4 supercharges). We now proceed to show that this interpretation is indeed correct. (For an analogous discussion for a D5-brane in a flat background, see [14].)

The charge $\tilde{U}_{\mu}$ transforms in the $(5,1)$ representation of $S O(5) \times S O(3)$. The BPS solution corresponding to it can be interpreted in space-time as a triple intersection of a D5-brane, a D3-brane and either

- an NS5-brane in e.g. the 45679 directions,

- a D1-brane in e.g. the 4 direction, or

- a D7-brane in e.g. the 1234567 directions.

These configurations indeed preserve $1 / 8$ supersymmetry. Let us e.g. illustrate the first one. A charge in the $(5,1)$ representation of $S O(5) \times S O(3)$ selects either 1 or (by hodge duality) 4 of the 5 worldspace directions 45678, and either none or 3 of the 123 directions.

\footnotetext{
${ }^{15}$ Note that from the symmetry group point of view, $Z_{9}$, being a singlet, could also be interpreted as a 0-brane inside the D5; however, since there is no 0-brane in the IIB theory, we conclude that $Z_{9}$ must be associated to a fundamental string in the 9th direction. (A D-string would not yield a BPS configuration.)
} 
Suppose we take it to select 4 worldspace directions, for instance 4567, and none among the 123. Then we would have to associate it to a 4-brane inside the D5-brane, but there is no 4-brane in the IIB theory. However, since the 9th direction is inert under the worldvolume symmetry group, we can assume that we have a 5-brane instead, extending along directions 4567 and 9. Hence, we end up with the interpretation of an NS5-brane along directions 45679 (a D5-brane along these directions would not be BPS).

Analogously, $\tilde{Z}_{[\mu \nu]}^{(\kappa \lambda)}$ corresponds to one of the following branes:

- a D5-brane in e.g. the 23456 directions,

- a D3-brane in e.g. the 145 directions, or

- an NS5-brane in e.g. the 23459 directions.

So far, we have interpreted the charges for the $a=1$ case. In the $a=0$ case, the same charges appear in the $\{Q, Q\}$ anticommutator, and there are many more in $\{Q, S\}$ and $\{S, S\}$. These do not represent other solutions. Indeed, as explained in the previous subsections, and as will be illustrated in appendix B, these charges are partners of those in $\{Q, Q\}$ in representations of the bosonic conformal algebra. This is similar to the special conformal transformations $K$, which are just partners of the translations $P$. In fact, the charges in the $\{Q, Q\}$ anticommutator constitute a maximal commuting subset.

\section{Conclusions}

In this paper we have extended the BPS method for branes propagating in a flat background to the case of branes in a non-trivial, curved background. This method has two complementary aspects.

On the one hand, the energy density is written as a sum of squares in a handy way. This allows the derivation of a BPS bound and the corresponding first order BPS equation.

On the other hand, the BPS bound is interpreted in terms of a central charge appearing in the maximally extended worldvolume supersymmetry algebra. This algebra is constructed by first analyzing the bosonic symmetries preserved by the vacuum solution and second constructing the most general anticommutator of the corresponding supersymmetry generators, including all possible central charges.

We have explicitly analyzed the case of a D5-brane in the background of a D3-brane, both in the near and non-near horizon cases. We have obtained in a unified way the BPS equations previously found in [11] and [13].

In the case of the near horizon geometry, the bosonic symmetries of the vacuum solution are $S O(2,1) \times S O(5) \times S O(3)$, the worldvolume algebra is $O S p\left(4^{*} \mid 4\right)$, and the corresponding maximally extended worldvolume superalgebra is $O S p(1 \mid 16)$, whose algebra contains 16 supercharges. The BPS bound is associated to a central charge, representing strings ending on the D5-worldvolume. Thus the existence of a BPS configuration preserving all 16 supercharges has been understood at the level of the worldvolume supersymmetry algebra.

For the non-near horizon case, the bosonic symmetries of the vacuum solution are $\mathbb{R} \times$ $S O(5) \times S O(3)$ and the maximally extended worldvolume supersymmetry algebra is a contraction of $O S p(1 \mid 8)$, which contains 8 supercharges. The charges appearing in the worldvolume algebra are interpreted in terms of multiple intersections of branes. 


\section{Acknowledgments}

We would like to thank Piet Claus for interesting discussions. B.C. and A.V.P. thank the Physics Department of the University of Barcelona for the hospitality during a fruitful visit in which a substantial part of this work was performed. D.M. is supported by a fellowship from Comissionat per a Universitats i Recerca de la Generalitat de Catalunya. This work was supported in part by the European Commission TMR programme ERBFMRX-CT96-0045, AEN98-0431 (CICYT), GC 1998SGR (CIRIT), NSF Grant PHY9511632 and the Robert A. Welch Foundation. 


\section{A Notations}

We use $\alpha, \beta, \ldots$ for spinor indices. Gamma matrices are then of the form $\left(\gamma_{\mu}\right)_{\alpha}{ }^{\beta}$, but indices may be lowered or raised using the charge conjugation matrix $\mathcal{C}^{\alpha \beta}$ and its inverse, which has lower indices: $\mathcal{C}^{\alpha \beta} \mathcal{C}_{\gamma \beta}=\delta_{\gamma}^{\alpha}$. This allows us to use NW-SE conventions for raising and lowering indices, e.g.: $\lambda^{\alpha}=\mathcal{C}^{\alpha \beta} \lambda_{\beta}$ and $\lambda_{\alpha}=\lambda^{\beta} \mathcal{C}_{\beta \alpha}$. When we write in the text $\gamma_{\alpha \beta}^{\mu}$, this is thus $\gamma_{\alpha}^{\mu \gamma} \mathcal{C}_{\gamma \beta}$.

\section{B Superalgebras and subalgebras}

In the main text we referred to various Poincaré, adS and conformal superalgebras, and their relations as subalgebras or contractions. Here we will repeat the standard relations and generalize them to the extended worldvolume algebras. To have a less trivial example than $S O(2,1)$ as in the main text, we treat the algebra of the D3 background, which is $\mathcal{N}=4$ supersymmetric in $d=4$.

The ordinary supersymmetries in that case are the 16 generators $Q_{\alpha}^{i}$ where $\alpha$ denotes the spinor index of $S O(3,1)$, and $i=1, \ldots 4$ is a vector index for $S U(4)$. We use chiral notations, i.e.

$$
Q_{\alpha}^{i}=\frac{1}{2}\left(1-\gamma_{5}\right)_{\alpha}{ }^{\beta} Q_{\beta}^{i}, \quad Q_{\alpha i}=\frac{1}{2}\left(1+\gamma_{5}\right)_{\alpha}{ }^{\beta} Q_{\beta i}
$$

such that complex conjugation can be done by raising or lowering the index $i$ (see e.g. appendix $\mathrm{A}$ in [22] for more explanations).

The super-Poincaré algebra has non-zero (anti)commutators

$$
\begin{aligned}
\left\{Q_{\alpha}^{i}, Q_{\beta j}\right\} & =\gamma_{\alpha \beta}^{\mu} P_{\mu} \delta_{j}^{i} \\
{\left[M_{\mu \nu}, M_{\rho \sigma}\right] } & =\eta_{\mu[\rho} M_{\sigma] \nu}-\eta_{\nu[\rho} M_{\sigma] \mu} \\
{\left[P_{\mu}, M_{\nu \rho}\right] } & =\eta_{\mu[\nu} P_{\rho]}, \quad\left[M_{\mu \nu}, Q_{\alpha}^{i}\right]=-\frac{1}{4}\left(\gamma_{\mu \nu}\right)_{\alpha}{ }^{\beta} Q_{\beta}^{i} \\
{\left[U^{i}{ }_{j}, Q^{k}\right] } & =\delta_{j}^{k} Q^{i}, \quad\left[U^{i}{ }_{j}, U^{k}{ }_{\ell}\right]=\delta_{j}^{k} U^{i}{ }_{\ell}-\delta_{\ell}{ }^{i} U^{k}{ }_{j},
\end{aligned}
$$

where the latter is the $U(4)$ automorphism group. Again on $U$ complex conjugation is done by raising or lowering indices, and the antihermiticity of the generators is then reflected in

$$
\left(U_{A}\right)_{i}{ }^{j} \equiv\left(\left(U_{A}\right)^{i}{ }_{j}\right)^{*}=-\left(U_{A}\right)^{j}{ }_{i} .
$$

The super-Poincaré algebra is the semi-direct sum of the solvable algebra with only $Q_{\alpha}^{i}$ and $P_{\mu}$ which is in the first line of $(\overline{\mathrm{B} .2})$, the Lorentz-algebra $S O(3,1)$, and the $R$-symmetry algebra $U(4)$.

Now we look to extensions with central charges. As explained in footnote 1, we use this terminology to denote generators which appear in the anticommutator of supersymmetries. These thus also contain generators which are neither the space-time symmetry generators, nor singlets under space-time symmetries, i.e. generators violating the Coleman-Mandula theorem [23]. We can add generators to the anticommutator of the supersymmetries:

$$
\begin{aligned}
& \left\{Q_{\alpha}^{i}, Q_{\beta j}\right\}=\frac{1}{2}\left(\left(1-\gamma_{5}\right) \gamma^{\mu}\right)_{\alpha \beta} P_{\mu} \delta_{j}^{i}+\frac{1}{2}\left(\left(1-\gamma_{5}\right) \gamma^{\mu}\right)_{\alpha \beta} Y_{\mu j}{ }^{i} \\
& (4,1)+(4,15) \\
& \left\{Q_{\alpha}^{i}, Q_{\beta}^{j}\right\}=\frac{1}{2}\left(1-\gamma_{5}\right)_{\alpha \beta} R^{[i j]}+\frac{1}{2}\left(\left(1-\gamma_{5}\right) \gamma^{\mu \nu}\right)_{\alpha \beta} Z_{\mu \nu}^{(i j)} \\
& 2(1,6)+(6,10) \text {, }
\end{aligned}
$$

where $[i j]$ denotes an antisymmetric tensor, $(i j)$ a symmetric tensor and $Y$ is a traceless tensor. $R$ is complex while for $Z$ the complex conjugates are the selfdual and antiselfdual 
components. We have taken at the right hand side all possible terms consistent with the symmetry, in total 136 bosonic generators. We have given their content in representations of $S O(3,1) \times S U(4)$. The algebra $O S p(1 \mid 16)$ has all the generators in (B.4). In that superalgebra the 136 bosonic generators have non-trivial commutation relations, namely those of $S p(16)$, and the fermionic generators have the commutation relations with the bosonic ones appropriate for a 16-representation. However, as extension of the super-Poincaré algebra, all these bosonic generators mutually commute, and commute with the supersymmetries. Thus one may regard that algebra as a 'contraction' of $O S p(1 \mid 16)$, where first all the fermionic generators are scaled with $x$, the bosonic ones with $x^{2}$, and then the limit $x \rightarrow \infty$ is taken. The full extended super-Poincaré algebra is as before the semi-direct sum of this algebra with the $S O(3,1)$ Lorentz-generators $M_{\mu \nu}$, and the $U(4)$ automorphism generators $U^{i}$. All generators have commutators with $M_{\mu \nu}$ and $U^{i}{ }_{j}$ as indicated by their indices.

In a superconformal extension, we should also include the special supersymmetries $S_{\alpha}^{i}$, appearing in the commutator $\left[K_{\mu}, Q_{\alpha}^{i}\right]=\left(\gamma_{\mu}\right)_{\alpha}^{\beta} S_{\beta}^{i}$. These have opposite chirality as $Q^{i}$, thus $S^{i}=\frac{1}{2}\left(1+\gamma_{5}\right) S^{i}$. The usual superconformal algebra has anticommutators between the fermionic generators:

$$
\begin{aligned}
\left\{Q_{\alpha}^{i}, Q_{\beta j}\right\} & =\frac{1}{2}\left(\left(1-\gamma_{5}\right) \gamma^{\mu}\right)_{\alpha \beta} P_{\mu} \delta_{j}^{i} \\
\left\{Q_{\alpha}^{i}, S_{\beta j}\right\} & =-\frac{1}{2}\left(1-\gamma_{5}\right)_{\alpha \beta} D \delta_{j}^{i}-\frac{1}{2}\left(\left(1-\gamma_{5}\right) \gamma^{\mu \nu}\right)_{\alpha \beta} M_{\mu \nu} \delta_{j}^{i}+\left(1-\gamma_{5}\right)_{\alpha \beta} U^{i}{ }_{j} \\
\left\{S_{\alpha}^{i}, S_{\beta j}\right\} & =-\frac{1}{2}\left(\left(1+\gamma_{5}\right) \gamma^{\mu}\right)_{\alpha \beta} K_{\mu} \delta_{j}^{i} .
\end{aligned}
$$

Here $\left(P_{\mu}, D, M_{\mu \nu}, K_{\mu}\right)$ are the generators of the conformal algebra $S O(4,2)$ and $U^{i}{ }_{j}$ generate $S U(4)$. This superconformal algebra is $S U(2,2 \mid 4)$. It can be truncated to the superPoincaré algebra given above by deleting the generators $S, D$ and $K$.

Again we can consider a larger algebra with all generators allowed in the supersymmetry commutators. It contains the anticommutator (B.4) and a copy of it with $Q$ replaced with $S$ and all the bosonic generators getting a partner in the same way as $K_{\mu}$ is the partner of $P_{\mu}$ in (B.5). The remaining anticommutators are

$$
\begin{aligned}
\left\{Q_{\alpha}^{i}, S_{\beta j}\right\}= & -\frac{1}{2}\left(1-\gamma_{5}\right)_{\alpha \beta} D \delta_{j}^{i}-\frac{1}{2}\left(\left(1-\gamma_{5}\right) \gamma^{\mu \nu}\right)_{\alpha \beta} M_{\mu \nu} \delta_{j}^{i}+\left(1-\gamma_{5}\right)_{\alpha \beta} U_{j}^{i}{ }_{j} \\
& +\frac{1}{2}\left(\left(1-\gamma_{5}\right)\right)_{\alpha \beta} Y^{\prime i}{ }_{j}+\frac{1}{2}\left(\left(1-\gamma_{5}\right) \gamma^{\mu \nu}\right)_{\alpha \beta} Y_{\mu \nu}^{\prime \prime}{ }_{j} \\
\left\{Q_{\alpha}^{i}, S_{\beta}^{j}\right\}= & \frac{1}{2}\left(\left(1-\gamma_{5}\right) \gamma^{\mu}\right)_{\alpha \beta}\left(R_{\mu}^{\prime}{ }^{[i j]}+Z_{\mu}^{\prime}{ }^{(i j)}\right) .
\end{aligned}
$$

The ones in the last anticommutator are complex. The names have been chosen such that the generators with primes combine with those of $\{Q, Q\}$ without primes and their partners in $\{S, S\}$ in representations of $S O(4,2)$. In this way we have in the bosonic algebra

$$
(15,1)(P, D, M, K)+[(1,15)+(1,1)](U)+2(6,6)(R)+(15,15)(Y)+(20,10)(Z)
$$

as decomposition under $S O(4,2) \times S U(4)$. The generators have all non-trivial commutation relations, as they form the algebra $S p(32)$. The superalgebra is $O S p(1 \mid 32)$.

\footnotetext{
${ }^{16}$ In fact, this case is special in that the trace of $U^{i}{ }_{j}$ is not part of the simple algebra. It can either be included as a central charge or as an automorphism. In the first case, this means that the trace of $U$ is included in (B.5), but then it is central, i.e. it commutes with the supersymmetries, and we should thus write

$$
\left[U^{i}, Q^{k}\right]=\delta_{j}^{k} Q^{i}-\frac{1}{4} \delta_{j}^{i} Q^{k} .
$$

Alternatively, the $U(1)$ factor does not commute with the supersymmetries, i.e. (B.2) remains unchanged, but then it should not appear in (B.5), where we should thus replace $U^{i}{ }_{j}$ by $U^{i}{ }_{j}-\frac{1}{4} \delta_{j}^{i} U_{k}^{k}$.
} 
Let us finish by giving an overview of all the algebras involved, starting from $O S p(1 \mid 32)$.

\section{Extended WV algebra WV algebra Supercharges}

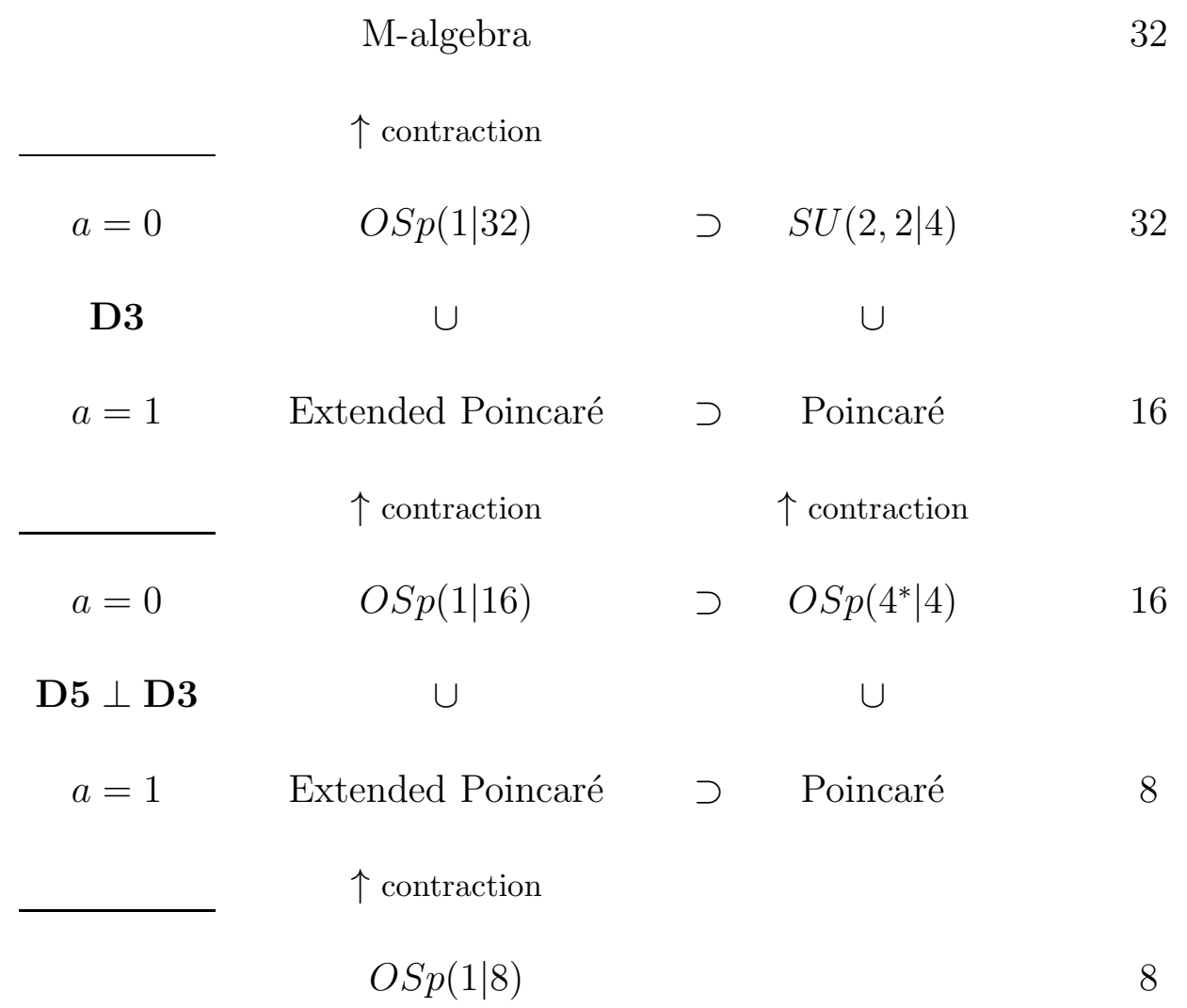

The contraction of this algebra with a factor $x$ for the fermionic generators, a factor $x^{2}$ for the bosonic ones and taking the limit $x \rightarrow \infty$ gives the $M$-algebra. Writing its generators as representations of the 4 dimensional Lorentz group and $U(4)$ we have the extended worldvolume algebra of the D3 background in the near-horizon case, containing the anticommutators in (B.4) and in (B.6). It has two important subalgebras. One is the non-extended worldvolume superconformal algebra $S U(2,2 \mid 4)$ containing the anticommutators in (B.5), the other is the extended super-Poincaré algebra, where the $S$-supersymmetries have been removed, together with parts of other $S O(4,2)$ multiplets. The non-extended super-Poincaré algebra is a subalgebra of the superconformal non-extended algebra as well as of the extended super-Poincaré algebra. Both these Poincaré algebras are contractions of simple algebras, respectively $O S p(1 \mid 16)$ and $O S p\left(4^{*} \mid 4\right)$, which are near-horizon algebras for the theory we consider in the paper, with a D5 worldvolume in a D3 background. The constructions explained above can be repeated in this smaller algebra, with a similar structure of subalgebras and contractions.

Remark that by taking other decompositions of the 32-component spinors we could, instead of going to $S U(2,2 \mid 4)$, also have gone to e.g. $O S p(8 \mid 4)$ for the conformal algebra in 3 dimensions, related to M2 or to $O S p\left(8^{*} \mid 4\right)$ for 6 dimensions, related to M5 [24]. The general setup of this article is also applicable to these cases.

\section{References}

[1] C.G. Callan and J.M. Maldacena, Brane dynamics from the Born-Infeld Action, Nucl. Phys. B513 (1998) 198-212, hep-th/9708147. 
[2] G.W. Gibbons, Born-Infeld particles and Dirichlet p-branes, Nucl. Phys. B514 (1998) 603-639, hep-th/9709027.

[3] P.S. Howe, N. D. Lambert and P.C. West, The self-dual string soliton, Nucl. Phys. B515 (1998) 203, hep-th/9709014.

[4] R. Haag, J.T. Łopuszańsky and M. Sohnius, All possible generators of supersymmetries of the S-matrix, Nucl. Phys. B88 (1975) 257.

[5] R. D'Auria and P. Frè, Geometric supergravity in $d=11$ and its hidden supergroup, Nucl. Phys. B201 (1982) 101;

H. Nicolai and A. Van Proeyen, Off-shell representations with central charges for 10dimensional super Yang-Mills theory, Nucl. Phys. B203 (1982) 510.

[6] J.W. van Holten and A. Van Proeyen, $N=1$ supersymmetry algebras in $d=$ 2,3,4, mod 8, J. Phys. A15 (1982) 3763. Nucl. Phys. B514 (1998) 603-639, hepth/9709027.

[7] E. Bergshoeff, J. Gomis and P.K. Townsend, M-brane intersections from worldvolume superalgebras, Phys. Lett. B421 (1998) 109-118, hep-th/9711043.

[8] J.P. Gauntlett, J. Gomis and P.K. Townsend, Bogomolnyi bounds for worldvolume solitons, JHEP 001 (1998) 033, hep-th/9711205.

[9] E.B. Bogomol'nyi, The stability of classical solutions, Sov. J. Nucl. Phys. 24 (1976) 449.

[10] E. Witten, Baryons and branes in anti de Sitter space, JHEP 07 (1998) 006, hepth/9805112.

[11] Y. Imamura, Supersymmetries and BPS configurations on anti-de Sitter space, Nucl. Phys. B537 (1999) 184, hep-th/9807179.

[12] A. Hanany and E. Witten, Type IIB superstrings, BPS monopoles, and threedimensional gauge dynamics, Nucl. Phys. B492 (1997) 152, hep-th/9611230.

[13] C.G. Callan, A. Güijosa and K.G. Savvidy, Baryons and string creation from the fivebrane worldvolume action, hep-th/9810092.

[14] E. Bergshoeff, G. Papadopoulos and J.P. van der Schaar, Domain walls on the brane, Phys. Lett. B430 (1998) 63-70, hep-th/9801158.

[15] T. Sato, The spacetime superalgebras from M-branes in M-brane backgrounds, Phys. Lett. B439 (1998) 12-22, hep-th/9804202.

[16] J.P. Gauntlett, N. D. Lambert and P.C. West, Supersymmetric Fivebrane Solitons, hep-th/9811024.

[17] P. Claus, R. Kallosh, J. Kumar, P.K. Townsend and A. Van Proeyen, Conformal theory of M2, D3, M5 and 'D1+D5' branes, JHEP 06 (1998) 004, hep-th/9801206.

[18] W. Nahm, Supersymmetries and their representations, Nucl. Phys. B135 (1978) 149.

[19] P. Claus, R. Kallosh and A. Van Proeyen, Conformal Symmetry on World Volumes of Branes, to appear in the proceedings of the 'Trieste Conference on Superfivebranes and Physics in $5+1$ dimensions', hep-th/9812066. 
[20] W. Nahm, V. Rittenberg and M. Scheunert, Classification of all simple graded Lie algebras whose Lie algebra is reductive, J. Math. Phys. 17 (1976) 1626, 1640;

V.G. Kac, A sketch of Lie superalgebra theory, Commun. Math. Phys. 53 (1977) 31.

[21] P.K. Townsend, M-theory from its superalgebra, in Strings, branes and dualities, NATO ASI series (Kluwer Academic Publishers, 1999), eds. L. Baulieu et al., p.141, hepth/9712004.

[22] A. Van Proeyen, 'Vector multiplets in $N=2$ supersymmetry and its associated moduli spaces', in 1995 Summer school in High Energy Physics and Cosmology, The ICTP series in theoretical physics - vol.12 (World Scientific, 1997), eds. E. Gava et al., p.256, hep-th/9512139.

[23] S. Coleman and J. Mandula, All possible symmetries of the $S$ matrix, Phys. Rev. 159 (1967) 1251.

[24] P. Claus, R. Kallosh and A. Van Proeyen, M 5-brane and superconformal (0,2) tensor multiplet in 6 dimensions, Nucl. Phys. B518 (1998) 117, hep-th/9711161. 